\title{
Les incidences spatiales de la grande vitesse
}

\author{
Une évaluation du projet «Swissmetro»
}

The objective of the research, referenced in the present article, was to try to evaluate the possible future spatial effects of the high-speed railway project "Swissmetro." The study is innovative from a methodological point of view, in that it constitutes an evaluation "ex ante" of the spatial effects of "Swissmetro."

Spatial effects are understood as impacts on the location of social and economic activities as well as on the location of households in Switzerland. In order to be able to develop the model, reference was made to the work of Plassard and Offner known for their evaluations "ex post" of the effects of the TGV in France. According to them, transport infrastructures have no direct or automatic effects but are produced by interaction with the social and spatial context into which the new transport service is to be inserted. 0 ur scenarios show that depending on this future context, "Swissmetro" will have very different impacts.

Connecting main Swiss urban centers will mainly influence the day-to-day functioning of Swiss cities and will have an impact on the future outlines of Swiss urbanization.

\section{Enjeux territoriaux liés a «Swissmetro»}

L'enjeu de cette recherche sur les incidences spatiales de «Swissmetro» menée dans le cadre du programme national de recherche 41 (PN R 41) «Transport et environnement» était double: il consistait d'abord à créer une méthodologie appropriée d'évaluation avant réalisation, ce en identifiant des critères quantifiables de l'incidence spatiale. Puis, il s'agissait de comparer les résultats obtenus à l'Institut de Recherche sur l'Environnement Construit de l'Ecole polytechnique fédérale de Lausanne (IREC; Daniel, Schuler, Bassand, Rumley) avec ceux réalisés par nos partenaires à l'Institut pour l'aménagement national, régional et local de l'Ecole polytechnique fédérale de Zurich (O RL; G ruber, Zbin- den, Schmid, 2000). Les deux équipes ont tra va illé selon deux méthodes d'évaluation différentes. L'O RL a traité l'incidence spatiale de «Swissmetro» à l'aide du logiciel «Tranus», tandis que l'équipe lausannoise s'est basée sur les critères mis en évidence par l'évaluation ex post des effets de la grande vitesse en France, en Allemagne et au Japon.

Dans cet article, nous présenterons donc brièvement les aspects techniques du projet «Swissmetro» et développerons la méthode d'évaluation et ses résultats. L'incidence spatiale mise en évidence nous conduit à mettre en perspective les objectifs actuels de l'organisation du territoire helvétique.

\section{1 «Swissmetro»: un projet à haute rupture technologique et spatiale}

La tension entre progrès technologique et incidence territoriale est à la base même du projet «Swissmetro». Plus «Swissmetro» permettrait un gain d'accessibilité élevé, plus il est intéressant au niveau technologique, tandis qu'au niveau spatial, sa capacité à produire des effets - recherchés ou non - s'en trouve augmentée.

Le constat à la base de cette innovation technologique est simple: en raison de la topographie, de la structure bâtie en corridor et des processus de décision politique en Suisse, la grande vitesse ne peut pas y être réalisée en surface. «Swissmetro» synthétise quatre nouvelles technologies (infrastructure souterraine, vide d'air partiel, propulsion par moteurs électriques linéaires, système de sustentation et de guidage magnétique) dont la combinaison représente une innovation radicale.

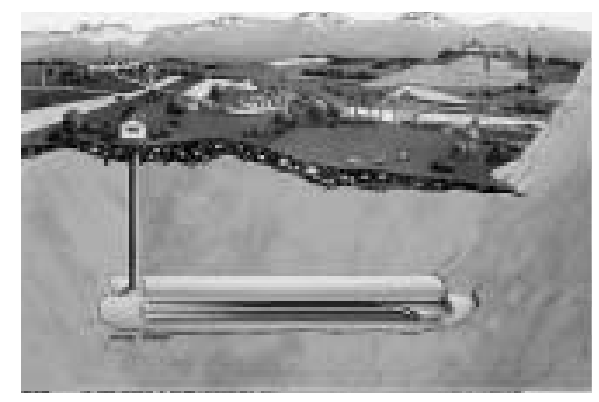

Fig. 1: Représentation de l'«intégration» de «Swissmetro» au paysage suisse, source Swissmetro SA, demande de concession, 1997

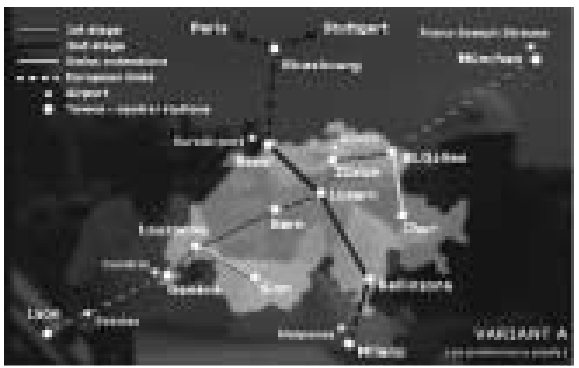

Fig. 2: Réseau «Swissmetro» variante $A$, Swissmetro SA

En raison des incertitudes technologiques liées à la combinaison de ces innovations, du coût de la réalisation d'un tel réseau et surtout de l'engagement actuel de la Confédération dans la mise en $œ u v r e$ de «Rail 2000», la mise en service de «Swissmetro» avant 2030 ne paraît pas réaliste. D'ailleurs, il n'existe pas encore de réseau officiel pour «Swissmetro», mais différentes variantes, dont une qui prolongerait les deux axes vers les grands centres voisins (cf. fig. 2).

La variante de base, évaluée par nos deux équipes de recherche est une adaptation de celle définie par Swissmetro SA. Elle consiste en deux axes principa ux qui se croisent à Lucerne. Les autres arrêts prévus sont G enève, Lausanne, Berne, Saint-G all et Lugano à l'emplacement des gares ferroviaires actuelles, tandis qu'à Bâle et Zurich, l'arrêt serait à l'emplacement des aéroports [1]. Dans notre évaluation, nous n'avons pas pris en compte les extensions éventuelles du réseau vers Sion et Coire [2]. Le choix du réseau est déterminant dans les incidences spatiales de la grande vitesse, étant donné la différenciation d'accessibilité qu'il entraîne.

1.2 Evaluer ex ante les incidences d'un projet de transport: les enjeux

Mettre en évidence la relation entre le développement territorial et la construction d'une nouvelle infrastructure de transport est un but caressé par tout porteur de projet de transport. Car parvenir à cet objectif, c'est assurer la légitimité d'un projet d'infrastructure nécessitant de lourds investissements financiers et un consensus politique fort. 
Depuis les recherches de Plassard et 0 ffner [3], les sciences sociales ne cessent de rappeler aux décideurs la difficulté de prévoir les effets des nouvelles infrastructures de transport à grande vitesse, les effets de celles-ci n'étant pas mécaniques mais liés à un contexte socio-spatial plus large. Ainsi la desserte par une infrastructure de transport est une condition nécessa ire mais pas suffisante du développement territorial. Les transports apparaissent plutôt comme un facteur du développement dans certains endroits et un vecteur de disparités spatiales dans d'autres.

$N$ otre recherche reprend l'hypothèse d'O ffner et Plassard selon laquelle l'incidence spatiale d'une infrastructure de transport est produite par interaction entre la nouvelle offre de transport et le contexte socio-spatial. L'incidence directe d'un mode de transport s'exerce uniquement sur les accessibilités, tandis que son incidence spatiale procède par une série de processus liés au contexte socio-spatial et n'est qu'indirecte.

\subsection{Les scénarios du développement territorial de la Suisse}

Evaluer l'incidence spatiale de "Swissmetro» consiste à comparer l'évolution $d u$ territoire Suisse avec et sans "Swissmetro». Compte tenu du délai de réalisation du nouveau réseau implique en premier lieu de modéliser l'évolution du système spatial suisse depuis 2000 jusqu'en 2050 sans «Swissmetro».

L'évolution du territoire suisse sans "Swissmetro» constitue une première recherche qui nous place déjà dans

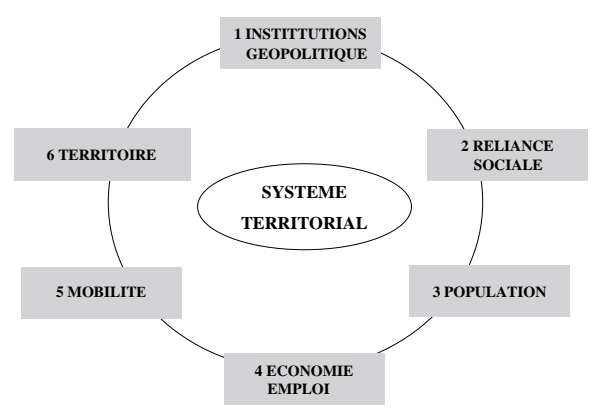

Fig. 3: Les six sous-systèmes du développement territorial

\begin{tabular}{|c|c|c|c|c|c|c|}
\hline $\begin{array}{l}\text { Sous- } \\
\text { systèmes/ } \\
\text { Scénarios }\end{array}$ & $\begin{array}{l}\text { Institutions } \\
\text { politiques }\end{array}$ & $\begin{array}{l}\text { Inégalités et } \\
\text { reliance } \\
\text { sociales }\end{array}$ & Population & $\begin{array}{l}\text { Economie et } \\
\text { emploi }\end{array}$ & Mobilité & Territoire \\
\hline $\begin{array}{l}\text { Une Suisse } \\
\text { riche et } \\
\text { solitaire }\end{array}$ & $\begin{array}{l}\text { Non-intégration } \\
\text { dans l'UE, } \\
\text { réformes internes } \\
\text { partielles }\end{array}$ & $\begin{array}{l}\text { Clivages sociaux } \\
\text { croissants, } \\
\text { tendances à la } \\
\text { ségrégation }\end{array}$ & $\begin{array}{l}\text { Croissance } \\
\text { élevée grâce } \\
\text { à une forte } \\
\text { immigration }\end{array}$ & $\begin{array}{l}\text { Forte croissance } \\
\text { et compétitivité, } \\
\text { marché d'emploi } \\
\text { dualisé }\end{array}$ & $\begin{array}{l}\text { Forte } \\
\text { augmentation } \\
\text { de l'offre }\end{array}$ & $\begin{array}{l}\text { Croissance } \\
\text { dispersée, } \\
\text { métropolisation } \\
\text { incohérente }\end{array}$ \\
\hline $\begin{array}{l}\text { Une suisse } \\
\text { dynamique } \\
\text { et ouverte }\end{array}$ & $\begin{array}{l}\text { Vent de } \\
\text { réformes, global/ } \\
\text { local compétitif }\end{array}$ & $\begin{array}{l}\text { Cohésion } \\
\text { métropolitaine }\end{array}$ & $\begin{array}{l}\text { Dynamique } \\
\text { démographique }\end{array}$ & $\begin{array}{l}\text { Stratégie de } \\
\text { performance }\end{array}$ & $\begin{array}{l}\text { Augmentation } \\
\text { contrôlée de } \\
\text { l'offre, nouvelles } \\
\text { régulations }\end{array}$ & $\begin{array}{l}\text { Métropolisation } \\
\text { maîtrisée, } \\
\text { croissance des } \\
\text { périphéries }\end{array}$ \\
\hline $\begin{array}{l}\text { Le scénario } \\
\text { tendance ou } \\
\text { le fleuve de } \\
\text { l'indécis }\end{array}$ & $\begin{array}{l}\text { Adhésion sans } \\
\text { vision }\end{array}$ & $\begin{array}{l}\text { Processus } \\
\text { d'individualisa- } \\
\text { tion }\end{array}$ & $\begin{array}{l}\text { Vieillissement de } \\
\text { la population }\end{array}$ & $\begin{array}{l}\text { Nouvelles } \\
\text { stratégies sans } \\
\text { garanties }\end{array}$ & $\begin{array}{l}\text { Croissance et } \\
\text { régulations non } \\
\text { coordonnées }\end{array}$ & $\begin{array}{l}\text { Périurbani- } \\
\text { sation continue }\end{array}$ \\
\hline $\begin{array}{l}\text { Développe- } \\
\text { ment } \\
\text { durable de } \\
\text { la Suisse }\end{array}$ & $\begin{array}{l}\text { Global et local } \\
\text { compatibles }\end{array}$ & $\begin{array}{l}\text { Solidarité } \\
\text { collective }\end{array}$ & $\begin{array}{l}\text { Croissance stable, } \\
\text { diminution à } \\
\text { moyen terme }\end{array}$ & $\begin{array}{l}\text { Une stratégie de } \\
\text { qualité de } \\
\text { l'emploi }\end{array}$ & $\begin{array}{l}\text { Régulation } \\
\text { conduisant à une } \\
\text { diminution de la } \\
\text { mobilité }\end{array}$ & $\begin{array}{l}\text { Maintien des } \\
\text { périphéries }\end{array}$ \\
\hline $\begin{array}{l}\text { Une Suisse } \\
\text { dodelinante } \\
\text { et isolée }\end{array}$ & $\begin{array}{l}\text { Rien ne change } \\
\text { et rien ne va, } \\
\text { réformes sous la } \\
\text { pression }\end{array}$ & $\begin{array}{l}\text { Le repli } \\
\text { identitaire }\end{array}$ & $\begin{array}{l}\text { Baisse démo- } \\
\text { graphique } \\
\text { considérable et } \\
\text { émigration }\end{array}$ & $\begin{array}{l}\text { Faible produc- } \\
\text { tivité, maintien } \\
\text { de branches } \\
\text { traditionnelles }\end{array}$ & $\begin{array}{l}\text { Diminution } \\
\text { quantitative, } \\
\text { perte de rentabi- } \\
\text { lité des réseaux }\end{array}$ & $\begin{array}{l}\text { Maintien relatif } \\
\text { des périphéries, } \\
\text { dégradation de } \\
\text { l'urbain }\end{array}$ \\
\hline
\end{tabular}

Fig. 4: Interactions entre sous-systèmes et scénarios

l'ordre des hypothèses. Afin de mieux cerner "le champ des possibles», cinq scénarios ont été réalisés. Ils représentent autant d'évolutions territoriales imaginables de la Suisse d'abord sans, puis a vec «Swissmetro».

Ces cinq scénarios:

- La croissance dynamique et ouverte.

- La Suisse riche et solitaire.

- Le scénario tendance ou le fleuve de l'indécis.

- La Suisse du développement durable.

- La Suisse dodelinante.

Les scénarios constituent cinq pistes d'évolution du système territorial suisse, système découpé pour les facilités de l'exercice en six sous-systèmes distincts selon fig. 3. Ce sont les interactions entre ces sous-systèmes qui conduisent à des évolutions contrastées du territoire suisse et de la mobilité qui s'y développe.

Les implications de ces scénarios sont observées à deux échelles territoriales:

Les cinq types d'urbanisation reflètent à la fois le clivage centre-périphérie et la dimension centres-couronnes (grands centres, centres moyens et petits, couronnes des grands centres, couronnes des centres moyens et petits, périphéries); les sept grandes régions (équivalents des régions NUTS-II d'Eurostat) montrent les disparités régionales (Région lémanique, Espace Mittelland, Suisse du Nord-O uest, Zurich, Suisse orientale, Suisse centrale, Tessin).
Les différences entre scénarios sont observées quantitativement à travers la répartition spatiale des emplois et des habitants dans les cinq types d'urbanisation ainsi que dans les sept grandes régions. Cette répartition est calculée à partir d'un modèle qui intègre les projections des trois scénarios de population de l'OFS de 1996 [4]. De même, l'incidence spatiale de «Swissmetro» sur la répartition des habitants et des emplois a été calculée en fonction de ces différentes entités territoriales.

\section{Effet spatial de «Swissmetro» en fonction des scénarios 2.1 Les processus de l'incidence spatiale de «Swissmetro»}

Pour intégrer «Swissmetro» dans les scénarios, il s'agit tout d'abord d'identifier les processus d'interaction entre grande vitesse et territoire. Des recherches théoriques et empiriques ont été menées tant en France, en Allemagne et qu'au Japon, pays où la grande vitesse a été réalisée et ses effets évalués ex post. Ces études mettent en évidence quatre paramètres déterminants à travers lesquels une infrastructure de transport conduit à des effets territoriaux:

- La congruence, c'est-à-dire l'adéquation entre la nouvelle infrastructure et les évolutions des besoins socio-spatiaux;

- Le gain d'accessibilité que procure la nouvelle offre de transport par rapport à l'existante; 

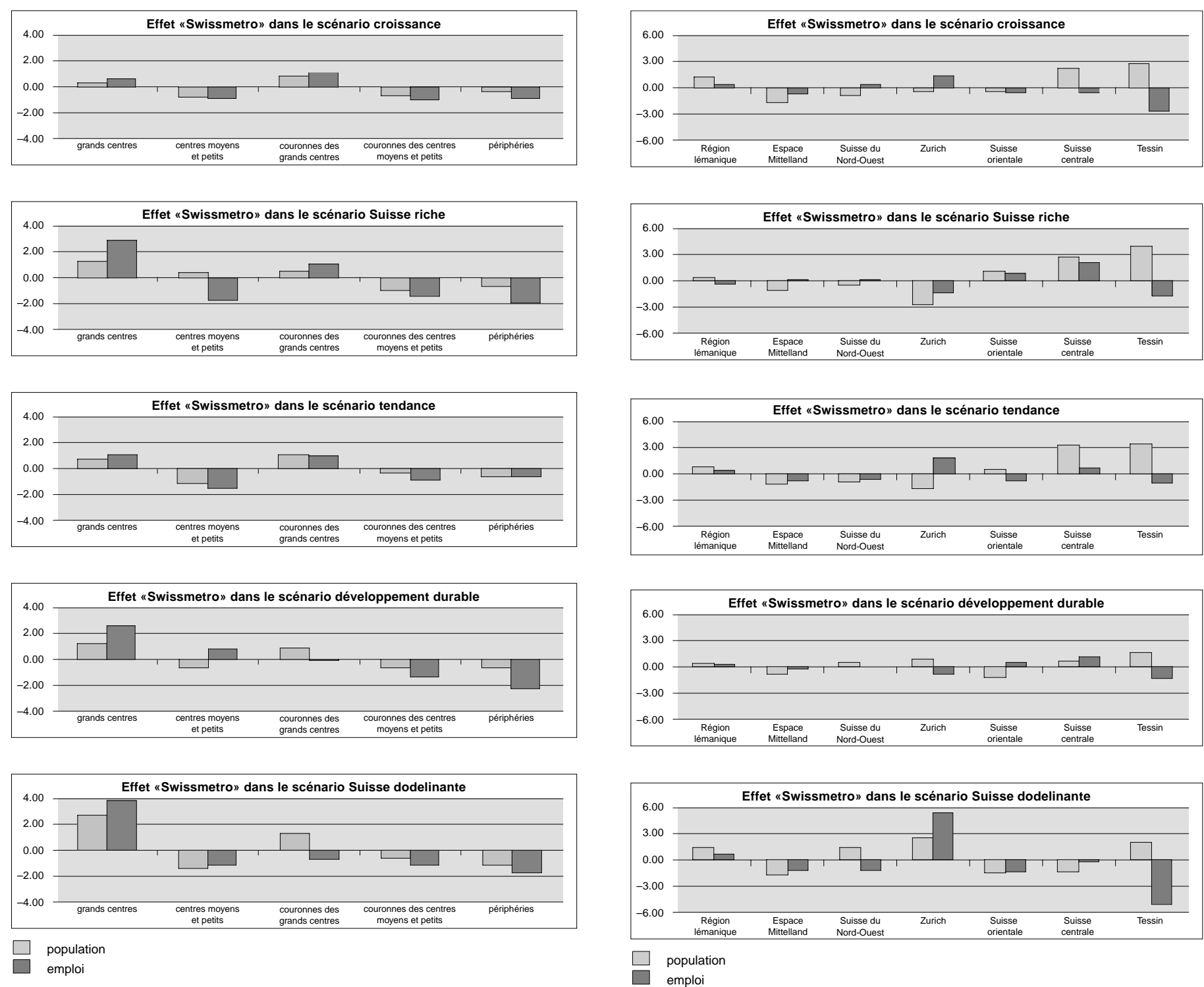

Fig. 5: Q uantification des effets de "Swissmetro» sur la répartition des emplois et habitants

- La capacité des territo ires à tirer parti de l'infrastructure (positionnement sur les marchés, concurrence entre territoires);

- Les stratégies d'acteurs (collectivités, acteurs économiques, individus) en amont (avant la réalisation) et en aval du projet (les utilisateurs de «Swissmetro»).

Ces quatre paramètres sont conditionnés par l'évolution du système territorial, autrement dit dans chacun des scénarios, l'impact spatial de «Swissmetro» se produit de manière particulière. A partir de ces quatre paramètres, nous avons quantifié l'impact de "Swissmetro» sur la répartition spatiale de la population, des emplois, ainsi que les flux pendulaires selon les cinq scénarios. Evaluer les effets spatiaux de "Swissmetro» suppose donc une connaissance fine du territoire et des interactions qui le dynamisent.
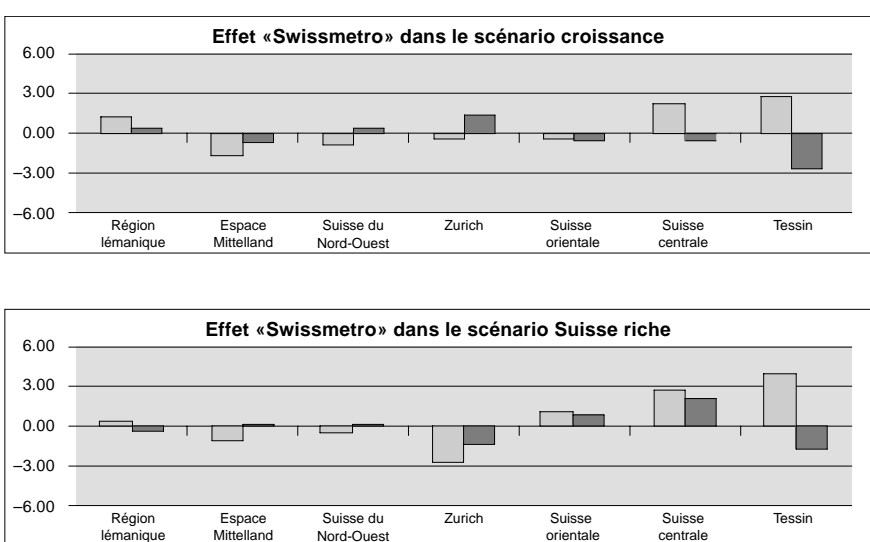

2.2 Résultats: l'incidence de «Swissmetro» dans les différents scénarios

Les résultats laissent apparaître que deux évolutions territoriales auront une influence majeure sur la capacité de «Swissmetro» à produire des effets:

- La densité et par là même les limites de capacité à une urbanisation plus a vancée des grands centres. Ce phénomène stimulant la spécialisation économique des métropoles transmet les effets de «Swissmetro» des villes-centres aux communes des couronnes (voir notamment la répartition de l'emploi dans les scénarios de la «Suisse riche et solitaire», de la «croissance dynamique et ouverte» mais aussi de la «tendance») [5]. Ce phénomène est particulièrement marqué dans les scénarios où la croissance économique est forte, où la dynamique démographique se maintient et où le coût des déplacements reste relativement bas.

- Les interrelations des métropoles suisses, selon que ces interrelations sont caractérisées par la position dominante de Zurich (scénarios «tendance», «Suisse riche et solitaire», "Suisse dodelinante») ou bien par une spécialisation économique complémentaire («croissance» et «durable»), les effets de «Swissmetro» sur la hiérarchie urbaine suisse seront différents. Dans le premier cas, les disparités entre grands centres seront renforcées, dans le second cas, un nombre plus large de grands centres suisses en profiteront, ce qui renforcera les grandes régions autour de ces centres [6].

Q uant aux espaces non desservis par le réseau «Swissmetro», ce n'est pas uniquement à cause de l'absence d'arrêts, c'est-à-dire de l'effet tunnel, qu'ils risquent de pâtir de "Swissmetro». Plus 


\begin{tabular}{|c|c|}
\hline Scénarios & Incidence spatiale de Sw issmetro \\
\hline $\begin{array}{l}\text { La croissance } \\
\text { dynamique et } \\
\text { ouverte }\end{array}$ & Favorise l'écart entre centres et périphéries mais ne creuse pas les écarts régionaux. \\
\hline $\begin{array}{l}\text { La Suisse riche et } \\
\text { solitaire }\end{array}$ & Renforce surtout the Greater Zurich Area. \\
\hline $\begin{array}{l}\text { Le scénario } \\
\text { tendance ou le } \\
\text { fleuve de l'indécis }\end{array}$ & $\begin{array}{l}\text { Davantage favorable aux grands centres internationaux, à la métropole lémanique et à } \\
\text { Zurich, plus qu'aux autres grandes agglomérations. }\end{array}$ \\
\hline $\begin{array}{l}\text { La Suisse du } \\
\text { développement } \\
\text { durable }\end{array}$ & $\begin{array}{l}\text { Soutient la reconversion et la densité dans les centres et diminue l'attractivité relative des } \\
\text { couronnes. Favorable à l'équilibre interrégional. }\end{array}$ \\
\hline $\begin{array}{l}\text { La Suisse } \\
\text { dodelinante }\end{array}$ & $\begin{array}{l}\text { Effet très centralisateur sur Zurich, seul pôle à même d'attirer le tertiaire supérieur dans ce } \\
\text { contexte économique marqué par la récession. }\end{array}$ \\
\hline
\end{tabular}

Fig. 6: Synthèse qualitative de l'effet «Swissmetro» en fonction des scénarios

encore que la desserte par la grande vitesse, c'est la spécialisation économique régionale qui gouverne la capacité d'un territoire à tirer parti de «Swissmetro»,

S'ils étaient desservis, les petits et moyens centres ainsi que les communes périphériques risqueraient de voir disparaître leurs services tertiaires supérieurs; c'est-à-dire les activités les plus sensibles à l'amélioration de la desserte par grande vitesse ferroviaire. La concurrence de ces services, fortement représentés dans les grands centres urbains serait difficilement soutenable pour les régions périphériques.

\subsection{Evaluation quantifiée ou quantitative des effets de «Swissmetro»?}

Avec I'ORL, nous avons comparé nos évaluations respectives des incidences de «Swissmetro» sur la répartition spatiale des emplois et de la population dans les scénarios «croissance» et «durable». Les deux équipes mettent en évidence que l'impact de l'infrastructure de transport varie en fonction du contexte des scénarios. Toutefois, les résultats obtenus par les deux méthodes distinctes ne se rejoignent que partiellement. II semble que certains paramètres à incidence spatiale sont difficilement intégrables au modèle "Tranus», comme les limites de capacité des territoires et les effets induits sur les périphéries d'une requalification des centres villes. Par contre, ce logiciel semble mieux adapté à évaluer les effets de différentes variantes de réseaux de transport et à les comparer entre elles.

\section{4 «Swissmetro» souligne les grands enjeux de l'organisation du territoire helvétique}

«A travers le projet «Swissmetro», l'aménagement du territoire acquiert l'opportunité de sensibiliser l'opinion à ses préoccupations et de lancer une discussion de fond» (Hutter, 1995). En voici les points principaux:

- Réalisé dans une seule logique économique, le projet «Swissmetro» risque de créer des effets pervers. II pourrait creuser les déséquilibres régionaux en Suisse en portant concurrence à d'autres systèmes de transport. Selon certains de nos scénarios, «Swissmetro» pourrait être réalisé de manière complémentaire aux autres systèmes de transport.

- «Swissmetro», tout en favorisant la mise en réseau des grands centres, relativise l'effectivité du concept de réseau des villes suisses, tel qu'il est formulé dans l'ouvrage de l'O ffice fédéral de l'aménagement du territoire «Les grandes lignes de l'organisation du territoire national» (1996). Ce principe d'organisation du territoire associe indistinctement les grands et moyens centres urbains suisses et les incite à fonctionner en réseau, tandis que «Swissmetro" mais également les logiques économiques - ont tendance à creuser les écarts entre les grands centres économiques et les autres agglomérations. Les différents nœuds du réseau «Swissmetro» devront se positionner les uns par rapport aux autres, «Swissmetro» entraîne surtout une spécialisation économique des centres desservis. L'effet de "Swissmetro», et de manière générale toute améliora- tion des moyens de transports, sont qualitatifs avant d'être quantitatifs. 0 r cette spécialisation économique engendre à son tour des besoins de déplacement croissants. Peut-on mettre en réseau des lieux sans entraîner une augmentation des déplacements entre ceux-ci?

- Enfin le projet «Swissmetro» alimente le débat sur les équilibres régionaux. La volonté d'équilibre entre régions centrales et périphériques en Suisse passe-til par des peréquations ou par une politique égalitaire rejetant tout projet susceptible de creuser les différences? SeIon la première option, "Swissmetro» peut jouer un rôle constructif dans l'organisation du territoire en Suisse, selon la seconde option, "Swissmetro» serait considéré comme un obstacle à l'équilibre régional.

Au regard de ces enjeux, nous conseillons de prendre la décision de réaliser ou non «Swissmetro» en fonction de l'analyse du contexte socio-spatial. Selon ces contextes illustrés par nos scénarios, le projet de la grande vitesse est nettement plus à même de favoriser des dynamiques spatiales souhaitables («scénarios croissance ouverte et dynamique», «développement durable» ma is aussi «Suisse dodelinante») que dans d'autres («Suisse riche et solitaire», «tendance»). II s'agirait alors d'instrumentaliser ce projet de transport pour stimuler les effets territoriaux souhaités. Un tel processus de mise en œuvre ne va pas sans une participation active des acteurs publics à la définition de ce projet de transport qui est par de nombreux aspects un véritable projet de société.

\section{N otes}

[1] L'équipe de l'O RL a également évalué l'incidence d'autres variantes de réseaux, notamment le croisement des deux tronçons à l'aéroport de Zurich-Kloten au lieu de Lucerne.

[2] Compte tenu des mécanismes de l'incidence spatiale de la grande vitesse, nous posons l'hypothèse qu'à un raccordement éventuel de Sion et Coire au réseau "Swissmetro» des mesures d'accompagnements devraient être préférées. En effet, elles auraient plus d'efficience sur le développe- 
ment de ces centres régionaux qui maintiennent leur position régionale dominante grâce à leur position périphérique.

[3] Entre autres publications Plassard F., Espace et dynamiques territoriales, textes rassemblés et présentés par P.-H. Derycke, Economica, 1992, pp. 243-26 et O ffner J.M., Les «effets structurants» du transport: mythe politique, mystification scientifique, L'espace géographique, Paris, 1993, n 3 , pp. 233-242.

[4] Voir la publication de l'O ffice fédéral de la statistique, Les scénarios de l'évolution démographique de la Suisse, 1995-2050, Berne, 1996.

[5] Ce phénomène explique les différences observées de l'effet de "Swissmetro» sur les cinq types d'urbanisation en fonction des scénarios envisagés.

[6] Ce phénomène explique les différences observées de l'effet de «Swissmetro» sur les sept grandes régions selon les scéna rios envisagés.

\section{Références}

BASSAND M., M étropolisation et inégalités sociales, Presses Polytechniques et Universitaires Romandes, Lausanne, 1997.

BUNDESAMT FÜR KONJUN KTURFRAGEN (éd.), Schweiz morgen, Vier Szenarien zur schweizerischen Zukunft, Berne, 1991.

CATTAN N . et al., Le système des villes européennes, collection Villes, anthropos, Paris, 1994.

DANIEL K., SCHULER M., BASSAND M., RUM LEY P.-A., "Swissmetro» et la Suisse en prospective, module F5a du PNR 41 «Transport et environnement», Berne, 2000.

GODET M., Manuel de prospective stratégique, tomes 1 et 2, Dunod, Paris, 1997.

GRUBER R., ZBIN DEN R., SCHMID W.A., Räumliche Effekte von "Swissmetro», M odellsimulation der Auswirkungen von Verkehrsinfrastrukturänderungen, N FP 41, Bern, 2000.

HUTTER R., Die Raumverträglichkeit der «Swissmetro», eine raumplanerische Annäherung, O RL-Bericht 94/ 1995, Zurich, 1995.

KAUFMANN V., Mobilité quotidienne et dynamiques urbaines, la question du report modal, Presses Polytechniques et Universitaires Romandes, Lausanne, 2000.

O FFICE FÉdÉRAL DE L'AM ÉN A G EM EN T DU TERRITO IRE, G randes lignes de l'organisation du territoire suisse, OCFIM, Berne, 1996.
O FFICE FÉDÉRAL DE LA STATISTIQ UE, Les scénarios de l'évolution démographique de la Suisse, 1995-2050, Berne, 1996.

OFFNER J.M., PUMAIN D. (sous dir. de), Réseaux et territoires, significations croisées, Editions de l'Aube, La Tour d'Aigues, 1996.

PEETERS D., THISSE J.F., THOMAS I., O n high speed connections and the location of activities, environment and planning, London, vol. 32, 2000

RO SSEL P. et al., Les enjeux des transports à grande vitesse, des méthodes pour l'évaluation des innovations technologiques, l'exemple de «Swissmetro», module F3 du PN R 41, «Transport et environnement», Berne, 1999. 